\title{
The Application of the Concept of Affordance to a Creative Design Method
}

\author{
Chien-Kuo Teng ${ }^{1,2}$ and Ming-Chuen Chuang ${ }^{2}$ \\ ${ }^{1}$ Department of Industrial Design, Shih Chien University, 70, Da-Zhi St. 104 Taipei, Taiwan \\ ${ }^{2}$ Institute of Applied Arts, National Chiao Tung University, \\ 1001 University Road, 300 Hsinchu \\ teng@mail.usc.edu.tw, cming@cc.nctu.edu.tw
}

\begin{abstract}
This research integrated ideas regarding affordance into a method for creative design, including five major steps: 1) Observe behavior; 2) Note down events happening or issues; 3) Figure out pattern; 4) Obtain messages of behavior perception; and 5) Reinforce message and naming. In this research, an "Another Hand" plate was designed to demonstrate the applicability of this method. In the application process, first, the researchers observe the dining environment, and discovered that it was difficult for people to scoop up the last few bits of food on the plate; therefore, they need the aid of extra tableware to finish their food. Then, observing users, we discovered that the spoon might turn around the plate. Next, we transform the idea into the design of the wedge to stop the food from moving. So the diner can finish the last bits of food using a natural eating motion. Lastly, with "Another Hand" plate as an example, we observed the process of 10 people eating with this plate. It was discovered that users could use this wedge to help scoop the food at $64.2 \%$ of the dining time. Hence, this invention won the final list design award of I.D.E.A. held in the USA in 2008. This research intends to review and revise this design method for better design for the reference of other inventors.
\end{abstract}

Keywords: affordance, observation, arousal, experience, design method.

\section{Introduction}

Product availability and usability are the primary key points during the process of industrial design. Via the product, it is necessary for designers to make users aware of appropriate user direction of the product and what the product can accomplish. Norman (1998) has pointed out that useful design is the criteria for future product competition. Over recent years, product designing has been emphasizing more on user experience and instinctive operation. Myerson (2001) stated that a new age of design, focusing on the whole experience of user interaction, required more subtle calibration. The development of psychology will also be studying and interpreting humans' subconscious behavior from another perspective, providing the product design more humanistic consideration in regards to the interaction between humans and objects. Of which, the concept of affordance evolved from ecopsychology has further expanded its view on humans' behavior to be the result as developed from the mutual influence 
between living things and environment, which is a perspective different from the concept of message handling as emphasized in the traditional cognitive psychology (Gibson, 1979; Mark, 1987; You, et al., 2007; Goto, 2008). Affordance is a direct perception theory created by eco-psychologist J.J. Gibson, which means the possibility that the environment endows upon a living thing's behavior. The concept itself is not a designing theory or method, but many designers today have extended it to application in the field of design. In the past, there has been study (Huang Rongcun, 1991) believing that Gibson's contribution is on the conceptual separation of imposed and obtained stimulation. Donald Norman (1998) used daily necessities to explain ways to strongly hint at an object's operation via affordance. For instance, a flat surface could be pushed, a round button could be turned, or a hole could be inserted. It could help designers during their product designing stage to enhance the products' availability and usability as they are being operated by their users, which would then allow users to more instinctively operate the products.

Attention to user's behavior during product operation has gradually become a designing trend around the world. Creation from product designer Naoto Fukasawa and the merchandise at MUJI have been widely accepted and enjoyed by many. Each believe that they have brought forth, such as "Without thought", "Later wow" and "What's happened naturally", has carried the following messages: user would ultimately understand product's design value via the behavior of implicit product operation; and the interaction between product and user could be made more natural. This point of view allows designers to have a more objective source of judgment when they ponder the interactive relationship between product and user as well as a perspective of wider vision and higher diversity. Thus, this research made the hypothesis on whether there could be a more concrete way or designing pattern, which can provide a foundation for designers to observe, step-by-step, the affordance that can be provided by the environment and then transform this observing experience in the proposal of an actionable designing result? Based on this goal, we are summarizing possible applicable methods for this affordance below, and we are also proposing a designing pattern and then applying the pattern in the design of a case study. We are hoping to build a preliminary design pattern that can stimulate design imagination based on practical design.

\section{Literature Review}

Zeisel (1984) believed that designing, an intricate activity, connects three basic activities: imagination, presentation and testing. And there are two categories of knowledge needed during designing according to their purpose: 1) knowledge that initiates a catalyst during imagination; and 2) knowledge that serves as test. (Korobkin, 1976; Zeisel, 1984) pointed out that parts of the designing knowledge are able to be observed via physical environment or environmental behavior. In regards to actual designing practice, designers would need to possess a sharp sense of touch towards the environment. IDEO founder Bill Moggridge proposed five designing steps: 1) understand, 2) observe, 3) visualize, 4) refine and 5) implement (Takeshi Goto, Masato Sasaki \& Naoto Fukasawa, 2008). Among these steps, understand and observe were identified as the top two elements in the designing process. Thus, the importance of these two steps is apparent. 
Affordance is the main concept under the direct perception theory of ecopsychology. Transforming the verb, afford, to a newly invented noun, affordance, James J. Gibson created this word to describe the specific relationship between living things and their surroundings (Gibson, 1979). Gibson believed that the messages that are influencing our perception and activity have already existed in the external world. And whether the observers could pick up these useful invariants would depend on observers' "purposeful" and proactive pursuit. The study explained the role "proactive discovery" plays in the process of searching for the invariants of form perception. Affordance has also been considered as one of the important designing factors in the enhancement of product usability and interactive capability (You \&Chen, 2007). In regards to the interpretation of "affordance", it has been interpreted with a following statement - "When the affordance of an object or the environment corresponds to the functions as expected, the design would exhibit with higher efficiency and easier use" (Lidwell, 2008).

Designers would also sometimes be accustomed to the existing method and thus be presenting ideas via the stereotypical or routine format. And they would thereby forget to observe the operation behavior that is actually taking place with a different attitude and find other potential opportunities to show off their design. The designer's subjective cognition, change of the environment's movement, and user's the behavior pattern would all influence the user's interpretation of the product language. Therefore, designers would need to pay attention to and consider the context within which users are using the products. This concept has been inspiring for the contemporary designers who have been seeking change. Designers are able to obtain a behavior's potential possibilities through observation. Via a designers' interpretation, they are able to rethink ways to operate instinctually and allow users to understand the operation via a form that is instinctive to the general public. As new vocabularies that are compatible with behavior are interpreted on the most apparent aspect of a product, the playfulness and the meaning of designing pieces would then be enhanced. Consequently, the comprehension gap between designer and user would also be reduced. This kind of proactive designing method is exactly the so-called perceptive message of reinforced behavior. Lastly, "design naming" would be used as a strategy to stir up user attention.

\section{Observation and Creation Methodology}

This research is planning to integrate the affordance concept as proposed in the field of psychology in the division of the environmental messages' observation methods. Moreover, we are also planning to discuss the interpretation method of affordance from a perspective of real case creation. We are trying to induce a more concrete creation principle and then proceed onto design creation with this methodology, so that we can provide designers a reference principle while they attempt to execute designing with the application of affordance. After going through literature organization and introduction of Chapter Two, we have obtained the three major characteristics of affordance that correspond to product design: 1) Utilize observation of the environment; 2) Activity-Centered Behavior Method; and 3) Point out perceptive messages symbolizing affordance. Then the three characteristics are further divided into five steps: 1) Observe behavior; 2) Note down events happening 
or issues; 3) Figure out pattern; 4) Obtain messages of behavior perception; and 5) Reinforce message and naming, so that we can further propose actual execution methods during the designing procedure. We have derived a preliminary procedure that applies affordance, which is displayed in Figure 1. We are explaining below in detail the process of applied affordance creation methodology as proposed in this research.

\begin{tabular}{|l|l|l|l|l|}
\hline \multicolumn{2}{|l|}{ Utilize Observation of the Environment } & $\begin{array}{l}\text { Activity-Centered } \\
\text { Behavior Method }\end{array}$ & Point out Perceptive Messages Symbolizing Affordance \\
\hline $\begin{array}{l}\text { 1. Observe } \\
\text { Behavior } \rightarrow\end{array}$ & $\begin{array}{l}\text { 2. Note Down } \\
\text { Events Happening } \\
\text { or Issues } \rightarrow\end{array}$ & 3. Figure out Pattern $\rightarrow$ & $\begin{array}{l}\text { 4. Obtain Messages of } \\
\text { Behavior Perception } \rightarrow\end{array}$ & $\begin{array}{l}\text { 5. Reinforce Message and } \\
\text { Naming }\end{array}$ \\
\hline $\begin{array}{l}\text { Observe the } \\
\text { environment for } \\
\text { behaviors that have } \\
\text { already happened, } \\
\text { the space or the } \\
\text { byproducts being } \\
\text { used. }\end{array}$ & $\begin{array}{l}\text { Examine ongoing } \\
\text { behavior. Then } \\
\text { break up the motion } \\
\text { during the activity. }\end{array}$ & $\begin{array}{l}\text { Record the physical } \\
\text { characteristics of the } \\
\text { material. Exhibit product } \\
\text { uniqueness of the new }\end{array}$ & $\begin{array}{l}\text { Obtain attributes and } \\
\text { apply: behavior comparison } \\
\text { and designing method to }\end{array}$ & $\begin{array}{l}\text { Name after examining } \\
\text { performance result or user } \\
\text { behavior after the change. }\end{array}$ \\
\hline
\end{tabular}

Fig. 1. The method of five steps and its flow chart to applying the concept of affordance

(1) Observe behavior: Designers need to observe the behavior in the environment that has already happened in the "past". Observation of the behavior that has already happened, space or byproducts that have been used include deterioration, remains or lack of signs (Zeisel, 1984). Deterioration refers to the wear resulted from frequent use, e.g. a small trail formed as people walk over the grass, or the slits left behind by knifes on a meat cutting board. Remains refer to things left behind from certain activities, e.g. the empty wine bottles after a banquet, cigarette ashes in the ash tray, opened food cans or scattered chairs. Through observation of the environment, events happening or issues are found. And no personal judgment shall be added during the observation so to avoid the influence from stereotypical image.

(2) Note down events happening or issues: Since activities are considered as a sequential process, videotaping or follow-up can be used to describe a complete activity procedure. Examine the behavior that is happening at the moment, including the relationship between objects and people as well as the relationship between objects and objects. Later, take apart the motions within the activity and examine further this "user habit".

(3) Figure out patterns: Designers can record the physical characteristics of a material, including shape, structure, hardness and surface pattern. Secondly, refer to people's existing user habits, e.g. trembling their feet while they ponder, unconscious hand motions while they dine, coordination of two hands' motion, etc. The information will be the base for the entry point where users begin their relationship with products so that the new design's product characteristics would be exhibited.

(4) Obtain messages of behavior perception: Obtain and apply observed signs of related behavior messages. The first application would be a comparison of similar behaviors. And the second application would be to accentuate or exaggerate the 
meaning displayed via a designing method (e.g. repetitive motions or objects that replace this motion).

(5) Reinforce message and naming: Through designers' naming per performance result or by describing the user behavior after transformation, designers can reflect on if the design is able to communicate the message they were hoping to pass on. Second, designers should consider the reminding effect that naming can place upon users. When users are using the product under specific circumstance, appropriate naming can help users to recall that special memory or experience of theirs. Thus the product can be made more easily accepted.

\section{Design Creation}

Taiwanese people eat mainly rice dishes, and fried rice is one of the most representative dishes in Taiwan within its rice eating culture. Fried rice is usually served in a shallow plate with wide mouth. We use it frequently, but we seldom notice where it needs to be improved. This design creation has been done via a planned designing pattern with "dining behavior design" as the theme (designer: Ying-Ting Kuo). During observation and actual practice of the procedure, we also discover different possibilities hidden within. We organize the designing steps and present the key points as below.

\subsection{Observe Behavior}

Designers noticed that there was often food left on the dining plate and thus found out this issue. Whether people were dining with chopsticks or spoons, or having food that was served on a plate, it was always difficult to finish that last bits of food. And when using other utensils to supplement the dining process, its purpose was exactly to assist the behavior motion of "stopping". A corresponding relationship exists between spoon/dining plate and people.

\subsection{Note Down Events Happening or Issues}

When we continued observing the actual dining process, we noticed that people would be accustomed to using another hand to hold other utensils (e.g. another spoon) to supplement their dining and finish the last bits of food. In terms of the reasons that make it difficult to scoop up the last bits of food, it could be that the food in the plate was reduced, the weight was also decreased. Correspondently, affordance that could stop the spoon to scoop up the food was also reduced. As a result, the food would be continuously pushed to the rim of the plate. We set a user behavior for our design according to the issues discovered at this stage: Don't overly change the original dining habit and allow users to finish their food from the plate more easily. Or in other words: Keep the form of the round-shaped ceramic dining plate, and find ways to increase the probability of finishing up the food. Sometimes there would be noise made when the spoon touches the rim of the ceramic plate or another supplemental utensil, which is also an indication for the users. 


\subsection{Figure Out Pattern}

Possible motions that could succeed in supplementing the goal of scooping up the food include: stopping, transferring and halting. According to the dining behavior observed above and the pre-set goal, we chose "stopping" to be our preliminary designing concept. Since the round-shaped dining plate originally possesses a shallow, thin and flat shape that is meant to hold and display food, it is not suitable to make any changes to its flat part. Also, because that last bits of food could not be stopped, they would be pushed to the rim of the plate. As a result, we had reason to think about our design starting from the plate rim. On the rim of the round-shaped dining plate, we designed a vertical convex stopping wall. When users found it difficult to scoop up the last bits of food, they would naturally push the food towards the convex stopping wall, which would help them to scoop up the food. Step 3 of our design creation ends here, and it has already provided a basic behavior possibility for the anticipated user behavior. In Step 4 that follows, we will be thinking about ways to reinforce the perceptive message or behavior image through further styling method. Besides allowing the anticipated operation to be established, the following step will also ensure our designing product has additional meaning.

\subsection{Obtain Messages of Behavior Perception}

In this step, designers started to think about related messages and signs of behavior that could further transform onto product's style and guide the users. In Step 1, it was observed that people have the habit of using another hand to supplement their dining process. This is a behavior that people have naturally developed in the environment. And following this universal behavior, signs of this behavior can serve as a clue for further styling transformation. Possible forms of signs include a hand and spoon, or the image of other utensils. Under the prerequisite of Step 2, we chose the image of a curved palm (Figure 2) to be the transformation of behavioral signs. With a fading surface, an invisible hand-like design is extended out along the rim of the roundshaped plate to help users during their dining process. The design corresponds to the events observed in Step 1 and places upon the product operation a more significant meaning. Thereby a new perceptive message was born.

\subsection{Reinforce Message and Naming - Another Hand}

Naming this design Another Hand corresponds to where the idea of this design comes from. We are hoping that users would realize the meaning of this image we have designed and the advantage of normal people having another hand to assist them during dining when they are near the end of their meal and have just discovered the behavior possibility hidden (using the convex stopping wall to stop the food).

\subsection{Design Result: Presentation of the Uniqueness of Another Hand}

The spotless white round-shaped ceramic dining plate has always been the best complement that brings out the arrangement of the food. However, the shallow and thin shape has often made it difficult for diners to finish every bit of the food when there are last bits of them left. Under the condition of not affecting the round-shaped 
dining plate, this design created a vertical wall to assist diners as they finish up last bits of food with our styling method. Even when the amount of food reduces, users can still push the portion of food left to the convex stopping rim. Through transforming the image of a curving palm to be the convex shape and naming it as Another Hand, it is as if an invisible hand is helping people to compete their meal. At the same time, it is also reminding us that we shall not waste a single bit of food. Considering user habits, the design keeps $70 \%$ of the opening arc area to allow both the left and right hands to operate smoothly. The angle at the convex stopping rim is easy for demolding manufacturing so that plates can still be piled up for storage. Figure 3 summarizes the designing thinking procedure described above.

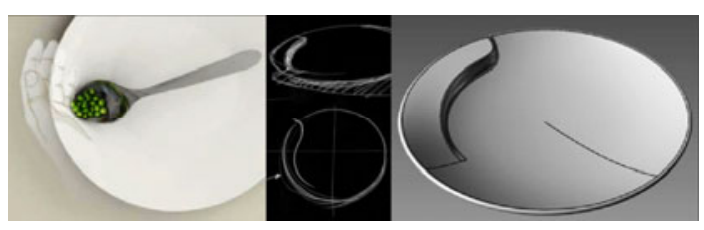

Fig. 2. Transformed image of hands to a concrete designing project.

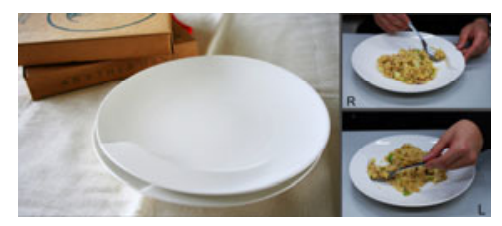

Fig. 3. Another Hand with opening arc area to allow both the left and right hands to operate smoothly, can piled up for storage.

\section{Design Result Assessment}

In regards to the creation result as brought forth by this research, we have performed an assessment via the two aspects of expert interview and user survey.

\subsection{Expert Interview Assessment}

In order to obtain more objective performance assessment for this research's creation result, this research invited two experts from the field of psychology and designing to provide our creation with some suggestions in the form of interviews. The expert from the field of psychology was a professor of Design Psychology at a college's dep. of industrial design. The expert from the field of designing was a senior designer with 12 years experience. The interview was separated into three parts: see, listen and interact. First, the expert individually observed the product without receiving any user instruction. Then, the designer described the circumstance and object for which the product was designed to. Creation though process was also explained. Lastly, interaction with experts was held for overall discussion. Organized below are the key comments that these two experts provided for this research's creation:

(1) Psychology Expert A: The work Another Hand provides a certain level of effective and appropriate affordance in terms of the food-stopping behavior. However, different results happen when encountering different food and utensils under the influence of different eating cultures. For example, when using a fork while having pasta, this stopping function might not be necessary. 
(2) Senior Designer B: This work chose the method of a vertical stopping surface to provide the food-stopping function, when the user is holding up the plate, the flat surface area at the rim of the vertical stopping surface can also provide the behavior possibility for the fingers to be placed on top of it. This will enhance the stability when the user holds up the plate and avoid the fingers from touching thee food served inside the plate.

\subsection{User Survey}

This research performed a user survey via the experimental method, where we observed how each subject finished the same portion of fried rice using spoon. There were a total of 10 subjects; each time the subject used the spoon to scoop up a spoonful of fried rice, a picture was taken. Following this procedure, we recorded down each user's behavior reaction and feedback towards the convex stopping part of Another Hand while they were having the fried rice. Since the speed of their dining process was not the focus of this research and to avoid any influence that timing might have on diner's habit, this experiment did not record the time it took for individual subject to finish eating. Each subject scooped up different amount of fried rice in each scoop. We defined $100 \%$ as the total number of scoops each individual took to complete the dining process from their first to their final scoop. Divided the total number of scoops by the number of scoops of fried rice picked up with the help of the convex stopping part, we could derive the progression ratio of the advantage of "used" convex stopping. Per the experiment result, we found that among the number of spoons it took for the 10 subjects to finish the fried rice, the minimum was 7 spoons and the maximum was 12 spoons. Some people made use of the convex stopping wall, as soon as $10 \%$, and some discovered the function as late as $90 \%$. And concluding the fried rice eating process for all 10 subjects, the overall average progression rate of discovering the function of and using the convex stopping part to assist in scooping up the fried rice was $64.2 \%$. Survey results of Another Hand users are summarized in Table 1 below.

Table 1. Summary of Another Hand Dining Plate Usage Survey Result

\begin{tabular}{|l|l|l|l|l|l|l|l|l|l|l|l|}
\hline Item Subject & $\mathbf{1}$ & $\mathbf{2}$ & $\mathbf{3}$ & $\mathbf{4}$ & $\mathbf{5}$ & $\mathbf{6}$ & $\mathbf{7}$ & $\mathbf{8}$ & $\mathbf{9}$ & $\mathbf{1 0}$ & Average \\
\hline Total Spoon Count & 10 & 10 & 10 & 7 & 12 & 10 & 11 & 8 & 10 & 8 & \\
\cline { 1 - 1 } $\begin{array}{l}\text { First Use of } \\
\text { Convex Stopping } \\
\text { Wall }\end{array}$ & 9 & 1 & 8 & 6 & 9 & 6 & 7 & 5 & 4 & 6 & \\
\hline Progression Ratio & $90 \%$ & $10 \%$ & $80 \%$ & $85.7 \%$ & $75 \%$ & $60 \%$ & $63.6 \%$ & $62.5 \%$ & $40 \%$ & $75 \%$ & $64.2 \%$ \\
\hline
\end{tabular}

Overall, there was no apparent difference between the performance of regular users and that of users with design-related background. We also discovered during the process that there were users who tilted the plate to increase the effectiveness of scooping up the fried rice. And there were also people who could use their left hand to use the design without any issues. Besides the scooping motion, we also observed users' unconscious motion of dangling their spoons while they chewed. Besides the 
motion of using the spoon to scoop up, there were also incidents where users were reminded of the function of convex rim as their spoon made a sound while it circled around the plate rim. The abovementioned observations all made it apparent that users were able to understand the hidden function of Another Hand.

\subsection{Brief Conclusion of Designing Results}

In terms of designing results, the work Another Hand participated in Taiwan's 1st Annual Universal Design Award in 2006 and received an Excellence Award. After enhancement was made on the design in 2007, i-use was authorized for its mass production, manufacturing and sales. In 2008, the work received the 2008 I.D.E.A. International Design Excellence Award of the U.S. A possible reason for the recognition of the work Another Hand lies in its fine integration between its functionality and the style symbolism of the behavior exhibited, which at the same time maintains the plate's original function diversity before the innovative design. Results of the user survey also showed that users might not notice the behavior possibility of the convex stopping part when they first started having the fried rice, but when the amount of fried rice was reducing, they would naturally push the fried rice towards the convex stopping part. And this phenomenon also corresponds to what the creator has originally anticipated. On the other hand, on the aspect of the development process of this research, it began from building a preliminary design of thought pattern to actual product manufacturing. And there were gradual adjustments on the organization and the writing of concept details as well as product details and pattern, which spanned a total of four years of development and revision. It has been apparent that the designing pattern is able to assist designer's thought process and possesses a preliminary application value.

\section{Conclusion}

This research proposed the three procedures of 1) observation of the environment; 2) activity-center behavior; and 3) connection with behavior image, along with five derived steps. Design case studies and evaluation were also used in the advanced discussion of the creation result. Even though its form of display was to bring the dining plate design to correspond to user's dining behavior and to enhance the convenience of the dining plate, there were still subjective differences during observation of the environment. After proactively figuring out the message, transformation was still needed to show off the existing affordance performance as a designed object. One thing worth mentioned is that the message mentioned here does not only direct to the consensus resulted from users "looking onto" the product, but it can also be viewed from a more expanded perspective to be a reflection of behavior, which is something that needs to be brought forth after a process of body contact. For the users, the message relates to that corresponds to affordance in the original environment after final design naming via the process of product operation. We are hoping future designers may discover more designing opportunities through the observation methodology described in this research. Listed below are four major conclusions and suggestions we have summarized from this research. 
1. In terms of the structure of designing pattern, affordance is originally a theory proposed by eco-psychology. Through discussion of related theories and documentaries as well as designers' creation experience, this research came to realize that "behavior" could be the main entry point from where it could find out a product design that corresponds to affordance. For the design creator, possessing a behaviororientated integrated advantage would assist designers during their designing process to create a style that would pass on the behavior message. Starting from planning product operation for the search of usable clues of affordance during the behavior procedure, product's usability, exterior design and reflective meaning would all be integrated step by step.

2. With regards to "The Design Procedure of the Applied Affordance Concept" as proposed by this search, even though it benefits designers in showing the structure of behavior message during their designing procedure, they still need to rely on their individual experience and aesthetics judgment during actual styling. Designing result can make the affordance design exceedingly explicit, and it can also make it utterly implicit. Overly exaggerated or poorly handled product messages can also create inappropriate association for the users that may cause errors during their operation.

3. Dining a plate design needs to take into consideration the size of the grains, the humidity and stickiness of the content (e.g. fried rice). These variables all influence the friction of the dining plate and change the method used to pick up the content inside the plate, and certainly they also influence the style of the product. Follow-up research can be based on the consideration abovementioned to integrate holding capability and unexpected effect as it looks for other applications.

\section{References}

1. Gibson, J.J.: The Ecological Approach to Visual Perception. Houghton Mifflin Company, Boston (1979)

2. Goto, T., Sasaki, M., Fukasawa, N.: The ecological approach to design. Azoth Books, Taipei (2008)

3. Huang, R.C.: The Psychology and Behavioral Researcher (1991) ISBN 957-09-0482-8

4. Krippendorff, K.: Design: A Discourse on Meaning. A Work Book. University of the Arts, Philadelphia (1994)

5. Lidwell, W., Holden, K., Butler, J.: Universal Principles of Design. UN Books, Taipei (2008)

6. Myerson, J.: IDEO: Master of Innovation. Laurence King Publishing, London (2001)

7. Norman, D.: Human-centered design considered harmful. ACM Digital Library Magazine (2005)

8. You, H.-c., Chen, K.-S.: Applications of affordance and semantics in product design. Design Studies 28 (2007)

9. Zeisel, J.: Inquiry by Design-Tools for Environment-Behavior Research. Cambridge University Press, Cambridge (1984) 